\title{
The Technology Revolution at the Limits of Detection
}

\author{
Philip Hexley ${ }^{1 *}$ and George Babcock ${ }^{2,3}$ \\ ${ }^{1}$ Cell Analysis Facility, University of Nebraska Medical Center, Omaha, Nebraska \\ ${ }^{2}$ Shriners Hospitals for Children, Cincinnati, Ohio.3Department of Surgery, University of Cincinnati, Cincinnati, Ohio
}

Received: November 15, 2013; Accepted: December 16, 2013; Published: December 19, 2013

*Corresponding author: Philip Hexley, Cell Analysis Facility, University of Nebraska Medical Center, Omaha, Nebraska; Tel: 402-559-6299; E-mail: phil. hexley@unmc.edu

\section{Editorial}

In 1980 Sydney Brenner proclaimed "Progress in science depends on new techniques, new discoveries, and new ideas, probably in that order", but this was not a novel concept. For decades people have understood the biosciences are not merely hypothesis-led but are driven as much by technological developments. New tools and technologies are essential in advancing modern biology, allowing lines of investigation that otherwise remain inaccessible.

One of these technological advances occurred over 40 years ago with the development of the flow cytometer. Flow cytometry is an increasingly widespread cellular analysis technique owing its popularity to the rapid, multi-parametric analysis capabilities whilst retaining information at the single cell level. Immunology is one of the focal areas revolutionized by this technology, allowing cells to be probed and sub-categorised by their cell surface phenotype and function.

One area which has recently found interest in wider areas of immunology is the analysis of extracellular vesicles, to understand the role they play in many systems. Not a new discovery, these extracellular vesicles have historically been identified as platelet dust in microscopy and rejected as cellular debris in flow cytometry [1,2]. However, sub-categories of these extracellular vesicles are now being identified as potential biomarkers.

Although nomenclature is used somewhat ambiguously in the literature, a review article by Gyorgy et al. [3] outlined the broad classification commonly accepted, defining three categories of extracellular vesicles originating from cells: exosomes $(<0.1 \mu \mathrm{m}$ diameter), microvesicles ( 0.1 - $1.0 \mu \mathrm{m}$ diameter), and apoptotic bodies $(>1.0 \mu \mathrm{m}$ diameter); each of which have different biological impact $[4,5]$. Exosomes are derived from exocytosis of intracellular multi-vesicular bodies, whereas both MV and apoptotic bodies arise from the plasma membrane of activated and apoptotic cells. The subject has been discussed in detail in an excellent review by Gutierrez-Vazquez et al. (2013).

Determining the role MVs play in disease states is of increasing interest in research and clinical settings as they contain cytoplasmic content and surface markers from the originating cell. Furthermore, the high mobility of MVs within the body implicates them as potentially important biomarkers in early disease states, and/or markers potentially indicative of treatment progression. Microvesicles are understood to be mediators in cell communication, through cytokine release and cell-interaction, to the degree of membrane-fusion leading to horizontal cell transfer of bioactive molecules. First documented functionally relevant in pre-eclampsia, microvesicles are now believed to play fundamental roles in a wide range of processes, including tumour progression and metastases, stem-cell expansion and renewal, coagulation, through to rheumatic diseases and inflammation [611].

At first glance using conventional flow cytometry (cFCM)for investigating the importance of the biology behind MVs appears to be an obvious extension of cellular analysis, due to the cellular origin of microvesicles and the same analysis requirements of as of cells. However, there are growing concerns using cFCM for analysis of MVs due to error-sources in sample collection through to physical limitations of detection.

Conceptually we cannot ignore the fact that cells and MVs are not one of the same thing, due to the drastic size difference between cells and MVs it is slowly becoming accepted that cFCM is not an appropriate technique for the analysis of MVs $[12,13]$. Although a remarkable technology, there are many concerns in $\mathrm{CFCM}$ which make this a suboptimal technique for analysis of MVs. Following is a brief outline of the greatest concerns currently facing small particle flow cytometry.

The use of polymer beads as a standardization is one of the most basic necessities, as without standardization confidence in data reproducibility is not possible. This polymer bead standardization becomes a fundamental concern as is it is understood that a bead may scatter up to 100 times more light than a MV, so if we can detect a 0.1 micron diameter polymer bead by light scatter it does not mean we can detect an equivalent size MV. This would imply that CFCM is not comprehensive in MV analysis but actually only analysing a proportion of the larger vesicles $[14,15]$.

Not just larger vesicles are being identified though, as another concern is over coincidence event detection. This is where small individual MVs would go undetected but due to close proximity in passing the optics of the flow cytometer, multiple MVs are acknowledged as a single event [16].

Finally from a technology standpoint, in cFCM we typically 
identify an event when light scatter is detected above the threshold in the forward scatter parameter. In small particle flow cytometry and microbial flow cytometry there are physical reasons for identifying events when light scatter is detected above the threshold in the $90^{\circ}$ angle (side scatter) parameter, but if such small particles do not create light scatter detected above the threshold then the option of identifying events by fluorescence is also available [17].

The combination of technology identifying fluorescence as a more sensitive detection, and the initial credence where due to the formation process that all MVs should have exposed phosphatidylserine (PS), studies suggested fluorescence would be a better parameter for identification through detection of fluorescently labelled Annexin V binding to the exposed PS. However it is undetermined whether fluorescence or light scatter is a better method for detecting MV events by FCM, as there is now convincing evidence that not all MVs are Annex in $\mathrm{V}$ positive [18]. Currently there is no all-encompassing MV-stain of which to identify events by fluorescence. So while some MV analysis would be more suited for fluorescence detection there would be sub-populations which may not be detected using this method. It may be that depending on the application this will determine if fluorescence or scatter detection is superior.

As hinted at above, technology capabilities are not the only concern in MV analysis, there are mounting concerns over protocol standardization and defining pre-analytical and analytical variables which impact the analysis also. These include sample collection: phlebotomy technique; needle gauge; position of tourniquet; sample handling: vortexing and centrifugation conditions; and potential false positives from confounding reagent particulate and antibody aggregates. This is of real importance as the manner in which these, what may appear to be trivial, protocol steps are carried out can lead to a significant change in MV numbers [19].

Currently there are no good answers to these analysis issues, to date there is no gold standard in MV analysis. There are many instrument developments which may lead to a gold-standard though, including modifications of cFMC such as optimizing detection angles of scatter collection $[20,21]$ and full spectrum detection leading to improving instrument sensitivity [22]. To further this, new instrumentation are being developed and tested for improved detection and high throughput, individual scrutiny of these extracellular vesicles [14,23,24].

Although certainly not comprehensive in detection capabilities, it appears correlations show CFCM is a useful tool in the analysis of MVs. Researchers are working on new and improved technologies to more accurately analyse particles of this size, but until a better standardis confirmed then researchers much step carefully in overstating their findings when analysing extracellular vesicles using conventional flow cytometry.

\section{References}

1. Wolf, P. (1967). The nature and significance of platelet products in human plasma. Br J Haematol, 13, 269-288.
2. O'Gorman M. R. G., \& Donnenberg, A. D. (2008). Handbook of Human Immunology. CRC Press.

3. Gyorgy, B., Szabo, T. G., Pásztoi, M., Pal, Z., Misjak, P., Aradi, B, et al. (2011). Membrane vesicles, current state-of-the-art: emerging role of extracellular vesicles. Cell Mol Life Sci, 68(16), 2667-2688.

4. Crescitelli, R., Lässer, C., Szabo, T. G., Kittel, A., Eldh, M., Dianzani, I., et al. (2013). Distinct RNA profiles in subpopulations of extracellular vesicles: apoptotic bodies, microvesicles and exosomes. J Extracell Vesicles, 2, 20677.

5. Akers, J. C., Gonda, D., Kim, R., Carter, B. S., \& Chen, C. C. (2013). Biogenesis of extracellular vesicles (EV): exosomes, microvesicles, retrovirus-like vesicles, and apoptotic bodies. J Neurooncol, 113(1), $1-11$.

6. Gutierrez-Vazquez, C., Villarroya-Beltri, C., Mittelbrunn, M., \& SanchezMadrid, F. (2013). Transfer of extracellular vesicles during immune cell-cell interactions. Immunol Rev, 251, 125-142.

7. Timar, C. I., Lorincz, A. M., Csepanyi-Komi, R., Valyi-Nagy, A., Nagy, G., Buzas, E. I., et al. (2013). Antibacterial effect of microvesicles released from human neutrophilic granulocytes. Blood, 121, 510-518.

8. Baj-Krzyworzeka, M., Baran, J., Szatanek, R., Mytar, B., Siedlar, M., \& Zembala, M. (2013). Interactions of human monocytes with TMVs (tumour-derived microvesicles). Biochem Soc Trans, 41(1), 268-272.

9. van der Pol, E., Böing, A. N., Harrison, P., Sturk, A., \& Nieuwland, R. (2012). Classification, Functions, and Clinical Relevance of Extracellular Vesicles. Pharmacol Rev, 64(3), 676-705.

10. Hargett, L. A, \& Bauer N. N. (2013). On the origin of microparticles: From "platelet dust" to mediators of intercellular communication. Pulm Circ, 3(2), 329-340.

11. Thery, C., Ostrowski, M., \& Segura, E. (2009). Membrane vesicles as conveyors of immune responses. Nat Rev Immunol, 9(8), 581-593.

12.Larson, M. C., Luthi, M. R., Hogg, N., \& Hillery, C. A. (2013).Calciumphosphate microprecipitates mimic microparticles when examined with flow cytometry. Cytometry A, 83(2), 242-250.

13. Robert, S., Poncelet, P., Lacroix, R., Raoult, D., \& Dignat-George, F. (2011). More On: Calibration for the Measurement of Microparticles: Value of Calibrated Polystyrene Beads for Flow Cytometry-Based Sizing of Biological Microparticles. J Thromb Haemost, 9(8), 16761678.

14.van der Pol, E., Hoekstra, A. G., Sturk, A., Otto, C., van Leeuwen, T. G., \& Nieuwland, R. (2010). Optical and non-optical methods for detection and characterization of microparticles and exosomes. J Thromb Haemost, 9(80), 2596-2607.

15. Alison X. X., Min-Yu S. S., Thomas, M., Kelli, L., Elizabeth, T., \& Fiacco T. A. (2012). Improved flow cytometric assessment reveals distinct microvesicle (cell-derived microparticle) signatures in joint diseases. PLoS One, 7.

16.van der Pol, E., van Gemert, M. J., Sturk, A., Nieuwland, R., \& van Leeuwen, T. G. (2012). Single vs. swarm detection of microparticles and exosomes by flow cytometry. J Thromb Haemost 10(5), 919-930.

17. Hexley, P., Robinson, C. T., Osterburg, A. R., \& Babcock, G. F. (2012). Circulating microparticles do not all share biophysical light scatter properties with immune complexes when analyzed by flow cytometry Blood, 120(7), 1528-1529.

18. Connor D. E., Exner, T., Ma D. D., \& Joseph, J. E. (2010). The majority of circulating platelet-derived microparticlesfail to bind annexin $\mathrm{V}$, lack phospholipid-dependent procoagulant activity and demonstrate 
greater expression of glycoprotein Ib. Thromb Haemost, 103(5), 1044-1052.

19. Gyorgy, B., Szabo, T. G., Pásztoi, M., Pal, Z., Misjak, P., Aradi, B., et al (2011). Membrane vesicles, current state-of-the-art: emerging role of extracellular vesicles, Cell Mol Life Sci, 68(16), 2667-2688.

20. Toxavidis, V., Tigges, J., \& Groglio, K. (2013). Microvesicle Detection and Cell Sorting. ISAC XXVIII Congress of the International Society for Advancement of Cytometry: CYTO conference poster 219/B98, San Diego CA.

21.Toxavidis, V., Tigges, J., \& Groglio, K., (2013). Flow Cytometric Standarization for the Analysis of Microparticles. ISAC XXVIII Congress of the International Society for Advancement of Cytometry: CYTO conference poster 299/B178, San Diego CA.

22. Stoner, S. \& Nolan, J. (2013). Flow Cytometric Analysis of Single Lipid Membrane Vesicles. ISAC XXVIII Congress of the International Society for Advancement of Cytometry: CYTO conference Parallel Session, San Diego CA.

23. Müller, G. (2012). Novel Tools for the Study of Cell Type-Specific Exosomes and Microvesicles. J Bioanal Biomed, 4(4), 046-060.

24. Garza-Licudine, E., Deo, D., Yu, S., \& Uz-Zaman, A. (2010). Dunbar W. B. Portable nanoparticle quantization using a resizable nanopore instrument - the IZON qNano ${ }^{\mathrm{TM}}$. Conf Proc IEEE Eng Med Biol Soc. 